\title{
Amplifier similariton fiber laser with nonlinear spectral compression
}

\author{
Sonia Boscolo, ${ }^{1}$ Sergei K. Turitsyn, ${ }^{1}$ and Christophe Finot ${ }^{2, *}$ \\ ${ }^{1}$ Aston Institute of Photonic Technologies, School of Engineering and Applied Science, Aston University, Birmingham B4 7ET, UK \\ ${ }^{2}$ Laboratoire Interdisciplinaire Carnot de Bourgogne, UMR 6303 CNRS-Université de Bourgogne, \\ 9 avenue Alain Savary, BP 47870, Dijon Cedex 21078, France \\ ${ }^{*}$ Corresponding author: christophe.finot@u-bourgogne.fr
}

Received July 5, 2012; accepted August 29, 2012;

posted September 12, 2012 (Doc. ID 172052); published October 30, 2012

\begin{abstract}
We propose a new concept of a fiber laser architecture supporting self-similar pulse evolution in the amplifier and nonlinear spectral pulse compression in the passive fiber. The latter process allows for transform-limited picosecond pulse generation, and improves the laser's power efficiency by preventing strong spectral filtering from being highly dissipative. Aside from laser technology, the proposed scheme opens new possibilities for studying nonlinear dynamical processes. As an example, we demonstrate a clear period-doubling route to chaos in such a nonlinear laser system. () 2012 Optical Society of America

OCIS codes: $140.4050,320.5540$.
\end{abstract}

Rapid recent progress in passively mode-locked fiber lasers is closely linked to new nonlinear regimes of pulse generation [1]. These include dissipative solitons and self-similar pulse (similariton) propagation in passive and active fibers [2-6]. Experimental interest in these nonlinear pulse-shaping regimes is strongly driven by the possibility of achieving pulse energy and peak power performances much higher than those of prior approaches and that can directly compete with solid-state systems. In the normal group-velocity dispersion (GVD) regime typical of such lasers, dissipation plays a key role in balancing nonlinear phase accumulations and in the pulse shaping. In particular, spectral filtering is critical to stabilizing the evolution of amplifier similaritons [3] , which have the remarkable feature of being a local nonlinear attractor in the gain fiber of the laser. The pulse's independence from the global cavity parameters entailed by this nonlinear attraction also allows for dispersionmapped fiber cavities with large anomalous net GVD [7], which can generate shorter pulses with higher energy than soliton operation at large anomalous GVD. From a purely scientific point of view, mode-locked fiber lasers provide convenient and reproducible experimental settings for the study of a variety of nonlinear dynamical processes thanks to the relative simplicity and precision of fiber-optic experiments. Therefore, this research area is interesting in its own right. Despite substantial research in this field, qualitatively new phenomena are still being discovered. In this Letter, we propose a new fiber laser design that takes advantage of the process of spectral compression arising from nonlinear pulse propagation in optical fiber [8]. We numerically demonstrate that both highly chirped amplifier similaritons and transform-limited picosecond pulses can be generated with the proposed scheme. Nonlinear spectral compression yields increased spectral brightness compared to direct spectral filtering.

We consider a loop fiber laser configuration as shown in Fig. 1. A 4 m-long segment of ytterbium-doped fiber (YDF) with normal GVD provides pulse amplification and supports similariton dynamics with nonlinear attraction. The gain fiber is followed by a saturable absorber
(SA) element and a large optical bandpass filter (OBPF) that improve the stability of the laser. After the filter, the pulse enters a dispersive delay line (DDL) - commonly realized with diffraction gratings in $1 \mu \mathrm{m}$ systems - that supplies anomalous GVD with negligible nonlinearity, and imparts a negative frequency chirp onto the positively (normally) chirped pulse as produced by the fiber amplifier. The negatively chirped, large-bandwidth pulse is then spectrally compressed in a $2.34 \mathrm{~m}$-long segment of single-mode fiber with normal GVD [normally dispersive fiber (NDF)]. Indeed, the frequency chirp induced by selfphase modulation in the fiber (equaling minus the first derivative of the intensity-dependent nonlinear phase shift) brings a frequency downshift of the leading edge and an upshift of the trailing edge of the pulse, i.e., a positive chirp. Consequently, for a negatively chirped input pulse the linear and nonlinear phases cancel each other, leading to a redistribution of long and short wavelengths toward the center wavelength and therefore to spectral compression instead of spectral broadening [8]. This results in a significant increase of the energy spectral density. This spectral compression process is the key feature of the laser setup proposed here. A narrow OBPF is used after the NDF (only) to suppress the spectral wings due to imperfect compression. Note that an initial transient of spectral compression has been implicitly observed in the gain segment of a dispersion-mapped amplifier similariton laser operating at anomalous net GVD [7].

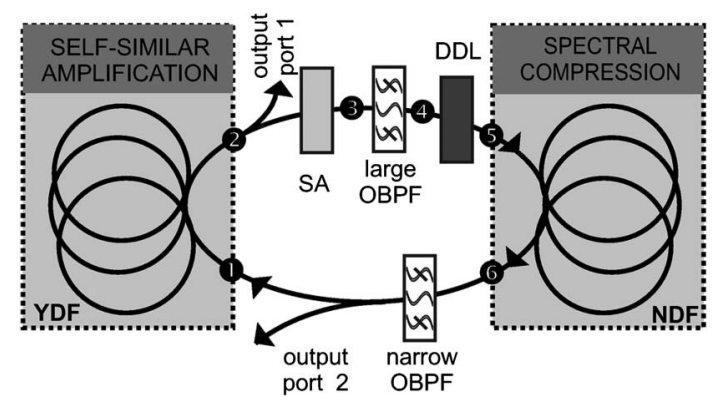

Fig. 1. Schematic of the laser. 
Propagation within each section is modeled with a modified nonlinear Schrödinger equation for the slowly varying pulse envelope:

$$
\psi_{z}=-\frac{i \beta_{2}}{2} \psi_{t t}+i \gamma|\psi|^{2} \psi+\frac{1}{2} g\left(\psi+\frac{1}{\Omega^{2}} \psi_{t t}\right),
$$

where $\beta_{2}=23 \mathrm{fs}^{2} / \mathrm{mm}$ is the GVD and $\gamma$ is the coefficient of cubic nonlinearity for the fiber section, with $\gamma=$ $0.0044(\mathrm{Wm})^{-1}$ for the YDF and $\gamma=0.0064(\mathrm{Wm})^{-1}$ for the NDF. The dissipative terms in Eq. (1) represent linear gain as well as a parabolic approximation to the gain profile with the bandwidth $\Omega$ corresponding to $40 \mathrm{~nm}$. The gain is saturated according to $g=g_{0} /\left(1+W / W_{0}\right)$, where $g_{0}=30 \mathrm{~dB} / \mathrm{m}$ is the small-signal gain, which is nonzero only for the gain fiber, $W=\int \mathrm{d} t|\psi|^{2}$ is the pulse energy, and $W_{0}=600 \mathrm{pJ}$ is the gain saturation energy determined by the pump power. The $\mathrm{SA}$ is given by a monotonically increasing transfer function $T=1-q_{0} /$ $\left[1+P(t) / P_{0}\right]$, where $q_{0}=0.8$ is the unsaturated loss, $P(t)$ is the instantaneous pulse power, and $P_{0}=150 \mathrm{~W}$ is the saturation power. The OBPFs are Gaussian transfer functions with $4.25 \mathrm{THz}$ and $0.25 \mathrm{THz}$ full width at half-maximum (FWHM) bandwidth, respectively. The DDL provides a total dispersion of $-0.197 \mathrm{ps}^{2}$. Linear losses of $60 \%$ and $84 \%$ are imposed after the amplifier and after the narrow filter, respectively, which summarize intrinsic losses and output coupling. The numerical model is solved with a standard symmetric split-step propagation algorithm, and the initial field is a picosecond Gaussian temporal profile.

The evolution is illustrated by plots of the root-meansquare (rms) pulse duration and spectral bandwidth, and of the pulse spectral profile as a function of position in the cavity (Fig. 2). Also shown is the evolution in an amplifier similariton fiber laser consisting of a YDF segment, an SA, and an OBPF [3]. The FWHM bandwidth of the filter is $2.5 \mathrm{THz}$ for this cavity, which is the minimum bandwidth allowing for stable mode locking, while other system parameters are the same as those used in our scheme. The pulse solution at different locations within the cavity is shown in Fig. 3. The pulse duration and bandwidth increase monotonically in the gain fiber as the pulse evolves toward the asymptotic attracting solution in the fiber, consistently with what is well known from self-similar propagation. A typical feature of amplifier similaritons is indeed that the pulses evolve toward a parabolic asymptotic solution: the pulse has a parabolic shape at the end of the gain fiber [Fig. 3(a)]. The associated spectrum exhibits some structure, as expected for a parabola with finite (though large) chirp. This pulse has a time-bandwidth product (TBP) of approximately 34 , and the output energy from port 1 is approximately $8.6 \mathrm{~nJ}$. Cancelation of the highly linear chirp outside the laser cavity can lead to high-quality temporally compressed pulses with durations as short as $100 \mathrm{fs}$ and peak powers well above $10 \mathrm{~kW}$.

In the similariton laser, the spectral filter compensates both the broad pulse duration and bandwidth after the gain segment [Fig. 2(b)]. Thus, the main pulse shaping mechanism relies on the attracting nature of amplifier similaritons, which is then compensated by strong
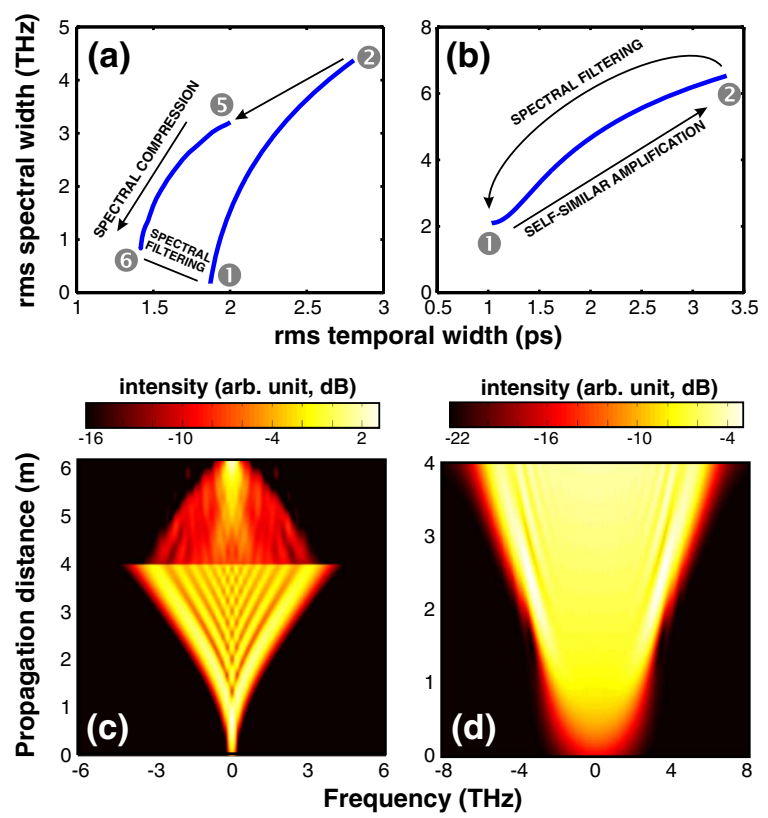

Fig. 2. (Color online) Phase diagram representing the combined evolution of the rms temporal and spectral widths of the pulse over one roundtrip in the cavity (a) with and (b) without nonlinear spectral compression. The locations in the cavity indicated in Fig. 1 are shown. Evolution of the pulse spectral profile along the cavity (c) with and (d) without nonlinear spectral compression.

spectral filtering. However, such filtering introduces high energy loss (about 75\%). In contrast, the pulse-shaping mechanism of the laser design proposed in this Letter is dominated by two nonlinear processes with distinctly
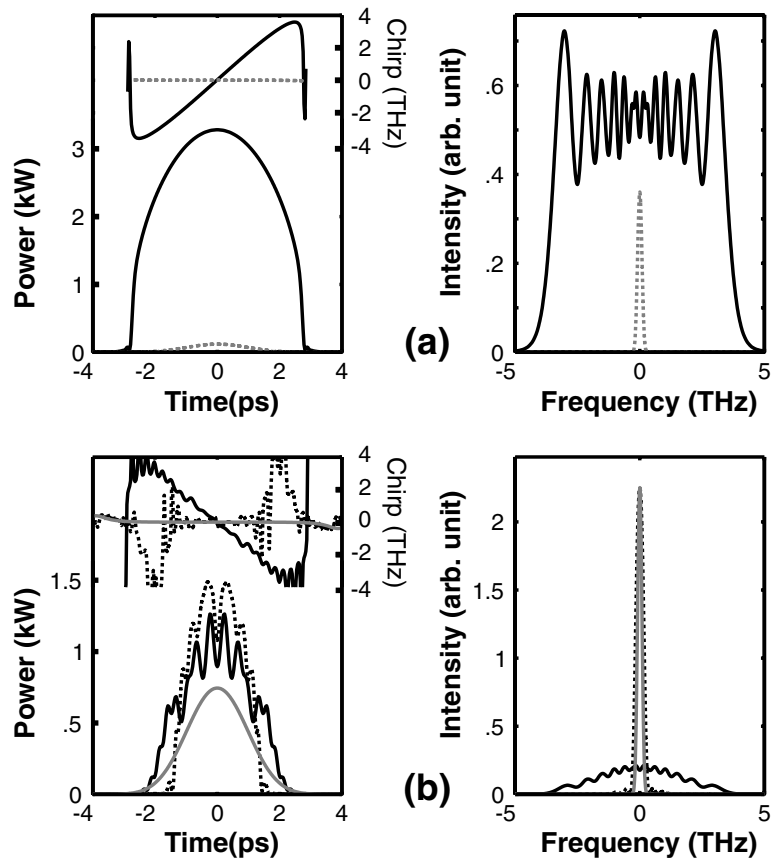

Fig. 3. Temporal intensity and chirp profiles (left) and spectral intensity profiles (right) of the pulse at (a) the entrance (location 1 in Fig. 1; dotted) and the exit (location 2; solid) of the gain fiber, and (b) the entrance of the passive fiber (location 5; solid black), the end of the passive fiber (location 6; dotted black), and after the narrow spectral filter (solid grey). 
different dynamics: similariton formation with nonlinear attraction in the gain fiber, and spectral compression arising from nonlinear propagation in the passive fiber and leading to large spectral narrowing [Fig. 2(a)]. The first large OBPF carves the output spectrum from the amplifier, and the linear DDL only affects the temporal width of the pulse. The compression factor, defined as the ratio of the spectral FWHM at the input and the output of the passive fiber, is approximately 11 and corresponds to an output FWHM of $0.35 \mathrm{THz}$ [Fig. 3(b)]. The latter is comparable to the bandwidth of the narrow OBPF. The energy spectral density of the pulse is therefore largely increased, and nonlinear spectral compression prevents strong spectral filtering from being highly dissipative. The output pulse from the spectral compression port (port 2) exhibits a transform-limited picosecond temporal profile with a TBP nearly equalizing the transform limit for a Gaussian pulse (0.441). Note that in the amplifier similariton laser the pulse after spectral filtering is still chirped and far from being transform limited, exhibiting a TBP of approximately 3 in the example considered here (Fig. 2). Transform-limited soliton pulses were generated in the anomalous-dispersion segment of a laser with similariton evolution in the amplifier [2]. However, the pulse energy scalability of the laser was limited by the soliton condition.

In the analysis so far, the overall dynamics of the laser cavity converged to a single-point attractor, with the pulse parameters at any given location within the cavity recovering the same values after every roundtrip. By increasing the length of the passive fiber segment above its $2.34 \mathrm{~m}$ reference value, a sequence of period-doubling bifurcations can be observed in the system [9], leading to chaotic pulse dynamics with the macroevolution of the pulse profiles never repeating. The laser shows the archetypal period-doubling bifurcation route to chaos. This is illustrated in Fig. 4 , which shows the pulse energy after the narrow filter as a function of the NDF length. Period$2,-4$, and -8 pulse solutions are seen, and burst into chaos occurs at $2.615 \mathrm{~m}$. At fiber lengths less than the reference value, the large anomalous residual chirp at the end of the fiber due to incomplete spectral compression connotes a significantly lower pulse energy.

In conclusion, we have numerically investigated a fiber laser design whose pulse-shaping mechanism relies on similariton formation with local nonlinear attraction in the gain segment of the oscillator and nonlinear spectral compression in the passive fiber. We have shown that nonlinear spectral compression allows for the direct

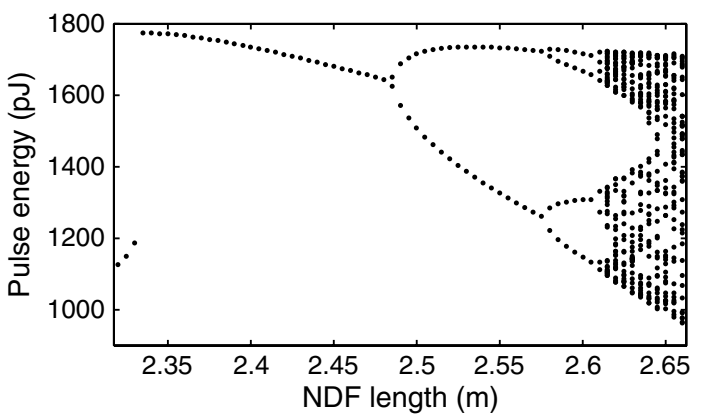

Fig. 4. One-dimensional Poincaré map: pulse energy at the output port 2 (Fig. 1) versus length of the passive fiber segment.

generation of transform-limited picosecond pulses from the laser, and benefits the laser's power efficiency by preventing strong spectral filtering from being highly dissipative. The new scheme for managing nonlinearities in a fiber laser cavity proposed here is radically different from established dispersion-managed schemes including anomalously dispersive elements, which entail small spectral breathing. The ability to generate highly chirped parabolic pulses and transform-limited spectrally compressed picosecond pulses from a single device will be attractive for applications. In particular, narrowspectrum pulses are desired in applications requiring high spectral resolution such as in nonlinear vibrational microscopy. By varying the length of the passive fiber, period-doubling bifurcations and chaotic system's behavior have also been reported.

\section{References}

1. W. H. Renninger, A. Chong, and F. W. Wise, IEEE J. Sel. Top. Quantum Electron. 18, 389 (2012).

2. B. Oktem, C. Ülgüdür, and F. Ö. Ilday, Nat. Photonics 4, 307 (2010).

3. W. H. Renninger, A. Chong, and F. W. Wise, Phys. Rev. A 82, 021805 (2010).

4. B. G. Bale and S. Wabnitz, Opt. Lett. 35, 2466 (2010).

5. C. Aguergaray, D. M'echin, V. Kruglov, and J. D. Harvey, Opt. Express 18, 8680 (2010).

6. A. Chong, H. Liu, B. Nie, B. G. Bale, S. Wabnitz, W. H. Renninger, M. Dantus, and F. W. Wise, Opt. Express 20, 14213 (2012).

7. W. H. Renninger, A. Chong, and F. W. Wise, Opt. Express 19, 22496 (2011).

8. S. A. Planas, N. L. Pires Mansur, C. H. Brito Cruz, and H. L. Fragnito, Opt. Lett. 18, 699 (1993).

9. L. Feng, P. K. A. Wai, and J. N. Kutz, J. Opt. Soc. Am. B 27, 2068 (2010). 